transfer was prematurity for the neonate, and respiratory failure for paediatrics.

Conclusion Optimizing and aligning practices between sending and receiving hospitals may improve inter-hospital handover efficiency and patient safety. Accurate recording of transfer data may be used to advocate expansion of the transfer service.

\section{P229 NAPKIN PSORIASIS-A REPORT OF TWO CASES}

Iva Topalusic*, Zdenka Plesa Premilovac, Nives Pustišek, Arnes Rešić. Children's Hospital Zagreb, Zagreb, Croatia

\subsection{6/archdischild-2019-epa.579}

Introduction Psoriasis is a T-cell mediated chronic inflammatory disorder of the skin. There are three age groups of pediatric psoriasis: infantile psoriasis, early onset psoriasis and pediatric psoriasis with psoriatic arthritis. Upper respiratory infection is the most common trigger of infantile psoriasis. We report two patients of early, Napkin psoriasis.

Case 1. A 5-months old female baby was reffered to our Emergency Department due to annular erythematous plaques in forehead, postauricular area, elbows, knees, back and diaper area. Due to suspected infection, ceftriaxon was introduced into therapy. Also, dexamthasone, mupirocin, and clotrimazole were localy applied. As therapy didn't show any clinical effect, punch biopsy was done and showed psoriasis vulgaris. Local therapy with aclomethasondipropionate was continued with good clinical response. A child was followed up during one year and no relapse was recorded.

Case 2. Nearly one-year old female baby was reffered to our Emergency Department after one week of antibiotical treatment of periorbital rash which was spread to the forehead, postauricular area, elbows, knees, back and diaper area. Napkin psoriasis was suspected and dexamethasone was localy applied. As it showed good clinal response, we concluded biopsy was not necessary. None of the children had signs of respiratory infection.

Conclusion Differential diagnosis of diaper dermatitis includes Napkin psoriasis, candidal diaper dermatitis and allergic contact dermatitis. In almost one third of all cases, psoriasis begins in childhood. Infants with psoriasis usually present with a diaper rash that is unresponsive to irritant diaper dermatitis treatment. Therefore all patients with diaper dermatitis should be properly evaluated and followed up.

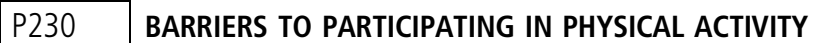 AND EXERCISE IN CHILDREN WITH TYPE 1 DIABETES MELLITUS (T1DM)}

Julie Evers, Madalene Khalil, Niall Dalton*, Orla Neylon, Paul Scully, Clodagh O'Gorman. University Hospital Limerick, Limerick, Ireland

\subsection{6/archdischild-2019-epa.580}

Aims Exercise and physical activity is an important component of a healthy lifestyle in all individuals with T1DM. However, despite efforts to promote an active lifestyle, a significant proportion of children with diabetes remain inactive and do not reach the recommended guidelines for exercise and physical activity. The aim of the current study was to examine barriers to undertaking physical activity in young people with T1DM.
Methods The study was a regional level observational clinical one within the University Hospital Limerick T1DM outpatient clinic, focusing on the perceived barriers to exercise and physical activity using the modified Barriers to physical activity in patient with Diabetes type 1 (BAPAD1) questionnaire. Questionnaires were completed between June and August 2018 and analysed using SPSS statistical software.

Results 55 children completed the survey. 55\% of respondents were female and age range was from 5-17 years, with all respondents been diagnosed a minimum of 2 years previously. $5 \%$ of respondents felt that having diabetes was a barrier to undertaking physical activity. Half of respondents stated that the risk of hypoglycaemia would be likely or very likely to be a barrier to undertaking physical activity. 29\% of respondents said that loss of control of their diabetes was not a barrier to undertaking physical activity. About 30\% of respondents felt that the risk of hyperglycaemia was a barrier to undertaking physical activity. $15 \%$ of respondents felt that their school schedule was a barrier to undertaking physical activity.

Conclusion The results from this study show that a high proportion of children felt that having T1DM was not a barrier to physical activity. The risk of hypoglycaemia was a significant barrier to activity. Future work should focus on addressing these barriers in more detail and the creation of guidance documents regarding overcoming such barriers.

\section{P231 EXPLORING PARENTAL KNOWLEDGE AND INFORMATION SOURCES PRIOR TO TYPE 1 DIABETES DIAGNOSIS TO INFORM FUTURE HEALTH PROMOTION CAMPAIGNS}

${ }^{1}$ Becky Hackett*, ${ }^{2}$ Edna Roche. ${ }^{1}$ The University of Dublin, Trinity College Dublin, Dublin, Ireland; '2Discipline of Paediatrics, The University of Dublin, Trinity College Dublin, Dublin, Ireland

\subsection{6/archdischild-2019-epa.581}

Introduction The diagnosis of Type 1 Diabetes (T1D) can be prolonged with delayed recognition of symptoms and delayed help seeking. Delayed diagnosis can result in the development of Diabetic ketoaciodosis (DKA), a potentially fatal metabolic decompensation resulting in a lasting trajectory of poor glycaemic control with increased risk of diabetes related complications. Health promotion campaigns designed to increase awareness of Type 1 diabetes have prompted earlier diagnosis and reduced the number of children and adolescents presenting in DKA at diagnosis in the majority of populations. Targeting of the media used in such health promotion campaigns would be anticipated to increase the effectiveness.

Aim To explore parental knowledge of T1D before their child's diagnosis, the pathway to diagnosis and parents most valued sources of health information to inform future health promotion campaigns.

Methods Parents of young children diagnosed with T1D attending a national patient and family support organisation event, completed a questionnaire exploring diabetes knowledge, symptoms, pathway to diagnosis and information sources.

Results Parents of twenty-five children participated. Median age at diagnosis was 5.0 (range 0.6-9.3) years. Median time since diagnosis was $1.8(0.1-10.25)$ years. Parents reported the classic T1D symptoms and suspected T1D in 56\% of cases. Pre-diagnosis $80 \%$ knew someone with T1D. Median duration 